\title{
LASTRAC.3d: Transition Prediction in 3D Boundary Layers
}

\author{
Chau-Lyan Chang* \\ NASA Langley Research Center, Hampton, VA23681
}

Langley Stability and Transition Analysis Code (LASTRAC) is a general-purpose, physics-based transition prediction code released by NASA for laminar flow control studies and transition research. This paper describes the LASTRAC extension to general three-dimensional (3D) boundary layers such as finite swept wings, cones, or bodies at an angle of attack. The stability problem is formulated by using a body-fitted nonorthogonal curvilinear coordinate system constructed on the body surface. The nonorthogonal coordinate system offers a variety of marching paths and spanwise waveforms. In the extreme case of an infinite swept wing boundary layer, marching with a nonorthogonal coordinate produces identical solutions to those obtained with an orthogonal coordinate system using the earlier release of LASTRAC. Several methods to formulate the 3D parabolized stability equations (PSE) are discussed. A surface-marching procedure akin to that for 3D boundary layer equations may be used to solve the 3D parabolized disturbance equations. On the other hand, the local line-marching PSE method, formulated as an easy extension from its 2D counterpart and capable of handling the spanwise mean flow and disturbance variation, offers an alternative. $A$ linear stability theory or parabolized stability equations based $\mathbf{N}$-factor analysis carried out along the streamline direction with a fixed wavelength and downstream-varying spanwise direction constitutes an efficient engineering approach to study instability wave evolution in a 3D boundary layer. The surface-marching PSE method enables a consistent treatment of the disturbance evolution along both streamwise and spanwise directions but requires more stringent initial conditions. Both PSE methods and the traditional LST approach are implemented in the LASTRAC.3d code. Several test cases for tapered or finite swept wings and cones at an angle of attack are discussed.

\section{Introduction}

Tnstability of laminar flow and transition to turbulence in a three-dimensional boundary layer such as a finite-span swept wing or axisymmetric forebodies at an angle of attack are important issues of modern aerodynamic designs. At cruise conditions, the drag caused by turbulent boundary layers over the surface accounts for a large portion of the total drag. Proper laminar flow control (LFC) to prevent or delay the onset of transition would significantly increase the vehicle performance and result in cost savings. The recent Defense Advanced Research Projects Agency (DARPA) effort on the Quiet Supersonic Platform (QSP) is aimed at developing technologies to achieve a low-boom, laminar flow design at supersonic speed [1]. In conjunction with the NASA and industry Supersonic Vehicle Technology (SVT) project, these transition-related efforts are geared towards the development of enabling technologies that will eventually lead to the next generation supersonic vehicles beyond Concorde. The core effort of these projects involves computational tool development and wind tunnel or flight experiments.

At NASA Langley Research Center, recent transition-related works have been focused on the development of a physics-based, integrated transition prediction framework that involves theoretical and numerical methods, prediction software development, and experimental investigations. This integrated tool consists of software that is designed to handle various stages of the transition process. The software encompasses a range of methods from the conventional $\mathrm{N}$-factor correlation (based on either the linear stability theory (LST) or the more advanced parabolized stability equations (PSE) approach) to simulations based on the absolute disturbance amplitudes. The Langley Stability and Transition Analysis (LASTRAC) software, made publicly available in 2002[2, 23], represents the first

\footnotetext{
*Research Scientist, Computational, Modeling, and Simulation Branch, email: Chau-Lyan.Chang@nasa.gov, Senior member, AIAA
} 
release of the integrated tool development. The code can be used for transition-related efforts for 2D or infinite swept wing configurations. This paper is focused on a major extension of LASTRAC to general 3D boundary layers.

Stability of 3D boundary layers has drawn much attention for several decades. Most of these investigations were based on the linear stability theory (e.g. Nayfeh [3], Cebeci \& Stewartson [4], and Malik \& Balakumar [5]) where stability analysis is done locally by assuming a slow-varying mean flow on the 3D surface. An eigenvalue problem similar to that in $2 \mathrm{D}$ boundary layers was formed in these analyses. $\mathrm{N}$-factor integration was then carried out to represent the disturbance growth. The computed $\mathrm{N}$-factor distribution on the 3D surface is thus closely related to the integration path chosen. Either the inviscid streamline or the group velocity line was commonly chosen to integrate the disturbance growth. Most of transition correlation results did not favor one over the other (see Malik \& Balakumar [5]). More recently, Atobe and Chen [6] also used a similar method for transition prediction on a Mach 2 natural laminar flow wing model.

The PSE method has been proven to be a viable alternative to traditional linear theory for $2 \mathrm{D}$ and infinite swept wing boundary layers. The PSE approach offers a very efficient marching solution that can account for the effects of boundary layer growth and nonlinear mode interaction. The capability of handling the latter effect forms the basis of a much more efficient method for absolute amplitude transition prediction without resorting to direct numerical simulations (DNS). The extension of PSE to general 3D boundary layers, however, is nontrivial due to several reasons. Firstly, unlike in the infinite swept wing boundary layers, the mean flow varies along the spanwise direction and this variation prevents the disturbance from being expressed as a simple Fourier decomposition in the spanwise direction. Therefore, a finite-difference description in physical space or a Fourier collocation scheme must be used to incorporate the changes along the spanwise direction. Secondly, proper disturbance boundary conditions are not easily identified in the spanwise direction except in the cases where periodic or symmetry conditions may be used (such as in an axisymmetric cone). Thirdly, because of the added degree of freedom on a 3D surface, the "streamwise" and "spanwise" directions are less apparent. As a result, a proper waveform is more difficult to construct as than its counterpart in a $2 \mathrm{D}$ boundary layer.

To circumvent the above difficulties, several strategies with varying degree of complexity may be employed. With proper disturbance boundary conditions, a plane-marching approach may be the most intuitive to use along a preidentified streamwise direction (e.g., along the free stream). In contrast to the $2 \mathrm{D}$ cases, the modal shape function is now a two-dimensional function. Given an initial distribution on a cross plane, the marching solution may be carried out. In the case where disturbance boundary conditions are difficult to find, a window or fringe function approach as suggested by Spalart and Watmuff [7] and Guo et al. [8] may be used to render a finite spanwise domain periodic such that a Fourier decomposition may be used. It should be noted that even with proper spanwise boundary treatment, additional issues such as the determination of a proper streamwise wave number also arise. Compared with $2 \mathrm{D}$ cases, this new approach is not as efficient because a cross-plane solution would require solving a largebandwidth matrix. Domain decomposition in conjunction with parallel processing or disk caching techniques are necessary to achieve more acceptable numerical efficiency.

For efficiency reasons, it is more desirable to have a simplified "local" approach that does not require a plane solution. Herbert [9] suggested a streamwise and spanwise surface-marching approach. In this method, two complex wave numbers are defined in accordance with two pre-defined spatial coordinates on a $3 \mathrm{D}$ surface. The evolution of the shape function is solved iteratively with two normalization conditions to determine two wave numbers. Initial conditions along two initial lines are required to start the marching process. The distribution of the disturbance growth may be obtained by integrating both wave numbers simultaneously on the surface and accounting for additional contributions coming from the shape function evolution. Because both wave numbers vary during the marching process, there is no clear definition of a particular disturbance mode. It appears that a growth pattern may be strongly related to the initial condition provided.

Alternatively, a local PSE approach may be constructed by locally varying the spanwise coordinate to align with a direction where the mean flow variation is minimal. Following the lead from the LST approach, a PSE marching solution along the inviscid streamline with locally varying spanwise direction offers a more economical method to account for $3 \mathrm{D}$ effects. In this paper, we formulate the stability problem by using a body-fitted nonorthogonal coordinate system that allows a flexible variation of spanwise coordinates along the marching direction. As will be discussed in the following section, mean flow and disturbance variation along the variable spanwise direction may be accounted for in this approach. In many applications, this spanwise variation does not play a significant role on 
the overall growth and may be neglected. In cases where substantial spanwise mean flow variation is present, the effect can be accounted for in this local analysis.

Both the surface-marching and local line-marching PSE methods for 3D boundary layers are investigated in the present study. These 3D PSE methods along with the more traditional LST-based approach are implemented into the new release of the LASTRAC.3d code. These different methods provide a means for cross validation. One of the main objectives of this paper is to justify the use of a local line-marching PSE approach for instability wave evolution in a 3D boundary layer. Because of its numerical efficiency, this local approach is recommended as an engineering tool for transition correlation. The flexible nonorthogonal coordinate system allows a varying spanwise direction that leads to better transition correlations. In this paper, we discuss the problem formulation for several 3D PSE methods. Some preliminary linear results for a transonic tapered wing, a finite 3D swept wing, and an axisymmetric cone at an angle of attack are included. Only linear results are presented in this paper. Transition prediction issues are assessed based on the disturbance amplification ( $\mathrm{N}$-factors).

\section{Problem Formulation}

To allow a more general waveform for 3D boundary layers. we use a nonorthogonal curvilinear coordinate system $\left(x^{1}, x^{2}, x^{3}\right)$ where $x^{1}$ and $x^{3}$ are along the boundary layer surface and $x^{2}$ along the wall-normal direction (see Fig. 1). Coordinates $x^{1}$ and $x^{3}$ are roughly aligned with the streamwise and spanwise directions, respectively and they are nonorthogonal in general. The Cartesian coordinate system is denoted by $(x, y, z)$. In the $\left(x^{1}, x^{2}, x^{3}\right)$ coordinate, the distance between two points infinitesimally separated in space may be evaluated by

$$
d s^{2}=g_{i j} d x^{i} d x^{j}
$$

where $g_{i j}$ is the fundamental metric tensor. The determinant of the matrix with an element of $g_{i j}$ is equal to the square of the Jacobian of the coordinate transformation. The contravariant metric tensor is defined according to

$$
g_{i k} g^{k j}=\delta_{i}^{j}
$$

where $\delta_{i}^{j}$ is the Kronecker delta and the tensor summation is implied. Both $g_{i j}$ and $g^{i j}$ can be evaluated based on the coordinate transformation from $(x, y, z)$ to $\left(x^{1}, x^{2}, x^{3}\right)$.

The Navier-Stokes equations in the curvilinear system can be written as (Aris [10])

$$
\begin{aligned}
& \frac{\partial \rho}{\partial t}+\left(\rho v^{i}\right)_{, i}=0 \\
& \rho\left(\frac{\partial v^{i}}{\partial t}+v^{j} v_{J}^{i}\right)=-g^{i j} p_{, j}+g^{i j}\left(\lambda v_{, k}^{k}\right)_{, j}+\left[\mu\left(g^{j m} v_{, m}^{i}+g^{i n} v_{, n}^{j}\right)\right]_{, j} \\
& \rho C_{p}\left(\frac{\partial T}{\partial t}+v^{j} T_{, j}\right)=\left(\kappa g^{j k} T_{, k}\right)_{, j}+\frac{\partial p}{\partial t}+v^{j} p_{, j}+\Phi
\end{aligned}
$$

where $\rho, p, T, \mu, \lambda, \kappa$ and $C_{p}$ are density, pressure, temperature, first and second coefficient of viscosity, thermal conductivity and constant pressure specific heat, respectively. The quantity $v^{i}$ is the contravariant velocity. The divergence of any vector is defined as 


$$
A_{, i}^{i}=\frac{\partial A^{i}}{\partial x^{i}}+\left\{\begin{array}{c}
i \\
i k
\end{array}\right\} A^{k}
$$

where the Christoffel symbol of the second kind is indicated by the curly bracket. The second-order tensor $v_{, j}^{i}$ is defined by

$$
v_{, j}^{i}=\frac{\partial v^{i}}{\partial x_{j}}+\left\{\begin{array}{c}
i \\
k j
\end{array}\right\} v^{k}
$$

The viscous dissipation function may be evaluated by

$$
\Phi=\left(\lambda v_{, j}^{i}\right)^{2}+\frac{1}{2} \mu\left(g^{j k} v_{, k}^{i}+g^{i m} v_{, m}^{j}\right)\left(g_{i n} v_{, j}^{n}+g_{j l} v_{, i}^{l}\right)
$$

For a Cartesian coordinate system, the metric tensor $g_{i j}$ reduces to

$$
g_{i j}=\delta_{j}^{i}
$$

For an orthogonal curvilinear coordinate system, the tensor becomes

$$
g_{i j}=h_{i}^{2} \delta_{j}^{i}(\text { no summation over } i)
$$

The quantity $h_{i}$ is the metric associated with the curvature along the $x^{i}$ direction. In either case, $g_{i j}$ becomes a diagonal tensor and the governing equations reduce to the conventional form. In general, $g_{i j}$ is a full (but symmetric) second-order tensor.

We decompose the dependent variables $\phi_{1}\left(\rho, v^{1}, v^{2}, v^{3}, T\right)$ into a steady-state mean part plus a perturbation,

$$
\phi_{t}\left(x^{1}, x^{2}, x^{3}, t\right)=\bar{\phi}\left(x^{1}, x^{2}, x^{3}\right)+\phi\left(x^{1}, x^{2}, x^{3}, t\right)
$$

If we substitute the above equation into Eq. (1) and subtract the steady-state equations, then we have the governing equations for the disturbances as

$$
\begin{aligned}
& \Gamma \frac{\partial \phi}{\partial t}+A \frac{\partial \phi}{\partial x}+ B \frac{\partial \phi}{\partial y}+C \frac{\partial \phi}{\partial z}+D \phi=V_{x x} \frac{\partial^{2} \phi}{\partial x^{2}}+ \\
& V_{x y} \frac{\partial^{2} \phi}{\partial x \partial y}+V_{y y} \frac{\partial^{2} \phi}{\partial y^{2}}+V_{y z} \frac{\partial^{2} \phi}{\partial y \partial z}+V_{x z} \frac{\partial^{2} \phi}{\partial x \partial z}+V_{z z} \frac{\partial^{2} \phi}{\partial z^{2}}
\end{aligned}
$$

Here, for convenience, we have replaced the coordinates $\left(x^{1}, x^{2}, x^{3}\right)$ with $(x, y, z)$. The coefficient matrices $\Gamma, A, B, C$, etc. are functions of all three coordinates for $3 \mathrm{D}$ boundary layers. They consist of a steady-state (functions of mean quantities) and time-dependent parts (functions of unsteady perturbations), e.g., 


$$
\Gamma=\Gamma^{l}(\bar{\phi})+\Gamma^{\prime \prime}(\bar{\phi}, \phi)
$$

The perturbation can be expressed in the following discrete Fourier series with a fundamental disturbance frequency $\omega$

$$
\phi(x, y, z, t)=\sum_{m=-M}^{M} \psi_{m}(x, y, z) e^{-i m \omega t}
$$

The governing equation for a single Fourier mode becomes

$$
\begin{aligned}
& A \frac{\partial \psi_{m}}{\partial x}+B \frac{\partial \psi_{m}}{\partial y}+C \frac{\partial \psi_{m}}{\partial z}+(D-i m \omega \Gamma) \psi_{m}=V_{x x} \frac{\partial^{2} \psi_{m}}{\partial x^{2}}+V_{x y} \frac{\partial^{2} \psi_{m}}{\partial x \partial y}+ \\
& V_{y y} \frac{\partial^{2} \psi_{m}}{\partial y^{2}}+V_{y z} \frac{\partial^{2} \psi_{m}}{\partial y \partial z}+V_{x z} \frac{\partial^{2} \psi_{m}}{\partial x \partial z}+V_{z z} \frac{\partial^{2} \psi_{m}}{\partial z^{2}}+F_{m}
\end{aligned}
$$

where the forcing function $F_{m}$ contains contributions from other Fourier modes due to nonlinear interaction and can be neglected for small disturbances. The coefficient matrices contain only the steady-state part $\left(\Gamma^{l}, A^{l}\right.$, etc.) and the time-dependent part ( $\Gamma^{n}, A^{n}$, etc.) has been moved to the right-hand side forcing function.

For a 2D or an infinite swept wing boundary layer, the basic flow is invariant along the spanwise direction. Periodic boundary condition in $\mathrm{z}$ is thus assumed. The mode shape function may be further decomposed into a discrete Fourier series,

$$
\psi_{m}(x, y, z)=\sum_{n=-N}^{N} \tilde{\psi}_{m n}(x, y) e^{i n \beta z}
$$

Substitute Eq. (6) into Eq. (5), we have a set of coupled partial differential equations (PDE) for $\tilde{\psi}_{m n}$ in two space dimensions, $(x, y)$. The derived equation is the harmonic linear Navier-Stokes (LNS) equation solved by Streett [11] and Guo et al. [12] using a large-matrix solver. Further assumptions based on scaling or parabolizing the derived equations may be made to simplify the solution procedure. In the PSE approach. a streamwise wave number is introduced to minimize the parabolizing error and to reduce the stringent resolution requirement in $x$. These procedures are discussed in the previous release of LASTRAC in details (Chang [2, 23]).

Two major issues stand in the way if we attempt to apply Eq. (6) directly for a full 3D boundary layer. Firstly, a periodic condition in $z$ is clearly invalid. In fact, proper boundary conditions are often difficult to identify in the spanwise direction. Depending upon the coordinate system used, there exists no clear physical distinction between $x$ and $z$ in some cases. For the ease of the following discussion, we assume that the coordinate $x$ is roughly aligned with the free stream direction and the spanwise coordinate (which may or may not be perpendicular to $x$ in general) may be chosen to align with a direction where the mean flow variation is minimal. The second issue is that for a given spanwise direction, the wave number $\beta$ is in general complex for a full $3 \mathrm{D}$ boundary layer. The mean flow variation in $z$ allows a disturbance mode to grow or decay in that direction. To overcome the above two issues, several methods with a varying degree of complexity may be used to solve Eq. (5) in a 3D space.

\section{A. Plane-Marching PSE}

In some cases, proper boundary conditions do exist in the spanwise direction. For example, periodic or symmetry boundary conditions may be applied in the azimuthal direction for axisymmetric configurations such as cones. Dirichlet or Neumann boundary conditions and no-slip conditions may be applied to the free stream and wall, respectively. 
Similar to the 2D cases, with a quasi-parallel assumption along the streamwise $(x)$ directions, we can rewrite $\psi_{m}$ as

$$
\psi_{m}=\widetilde{\psi}_{m}(y, z) e^{i \alpha_{m} x}
$$

where the streamwise wavenumber $\alpha_{m}$ is a function of the disturbance frequency according to the dispersion relation $\alpha_{m}=\alpha_{m}(m \omega)$. The linearized governing equation becomes

$$
\widetilde{B} \frac{\partial \tilde{\psi}_{m}}{\partial y}+\widetilde{C} \frac{\partial \tilde{\psi}_{m}}{\partial z}+\widetilde{D} \tilde{\psi}_{m}=V_{y y} \frac{\partial^{2} \tilde{\psi}_{m}}{\partial y^{2}}+V_{y z} \frac{\partial^{2} \tilde{\psi}_{m}}{\partial y \partial z}+V_{z z} \frac{\partial^{2} \tilde{\psi}_{m}}{\partial z^{2}}
$$

With homogeneous boundary conditions on the $y-z$ plane discussed above, a $2 \mathrm{D}$ eigenvalue problem similar to that investigated for secondary instability of stationary crossflow instability by Malik et al. [13] may be formed with $\widetilde{\psi}_{m}(y, z)$ as the $2 \mathrm{D}$ eigenfunction. Solving a $2 \mathrm{D}$ eigenvalue problem is rather time-consuming even with a moderate grid size on the $y$-z plane. Numerical efficiency may be significantly improved by using Arnoldi's method as was done by Lin and Malik [14] for the instability of the attachment line boundary layer.

To further account for the nonparallel effect, we may introduce a streamwise varying wavenumber, $\alpha_{m}(x)$, such that

$$
\psi_{m}=\tilde{\psi}_{m}(x, y, z) e^{i \int_{x_{0}}^{x} \alpha(5) d \xi}
$$

The governing equations become

$$
\widetilde{A} \frac{\partial \tilde{\psi}_{m}}{\partial x}+\widetilde{B} \frac{\partial \tilde{\psi}_{m}}{\partial y}+\widetilde{C} \frac{\partial \tilde{\psi}_{m}}{\partial z}+\widetilde{D} \widetilde{\psi}_{m}=V_{y y} \frac{\partial^{2} \tilde{\psi}_{m}}{\partial y^{2}}+V_{y z} \frac{\partial^{2} \tilde{\psi}_{m}}{\partial y \partial z}+V_{z z} \frac{\partial^{2} \widetilde{\psi}_{m}}{\partial z^{2}}
$$

where additional terms associated with the shape function derivatives in $x$ are introduced as compared to Eq. (8). Similar to its counterpart in 2D cases, Eq. (10) may be solved by a plane-marching procedure in $x$ provided that initial conditions are given on a cross plane. Unlike in 2D cases, determining the streamwise wave number $\alpha_{m}$ remains an issue. The introduction of the streamwise wave number $\alpha_{m}$ in the PSE approach is, primarily, to resolve the oscillatory (fast scale) part of the instability wave. Therefore, a constant $\alpha_{m}$ for the whole $(y, z)$ plane becomes inadequate when the disturbance mode structure varies substantially on the plane. We can make $\alpha_{m}$ a function of $x$ and $z$. The distribution of the streamwise wave number must then be determined by a normalization procedure local to the spanwise location $z$.

For flow configurations such as a finite 3D wing where periodic conditions are not applicable, a window function may be applied at the spanwise boundaries as suggested by Guo et al. [8]. The window function renders both the mean flow and disturbances periodic along the $z$ direction. Given a fundamental spanwise wave number, a single Fourier series may be used to represent both the mean flow and disturbances simultaneously. In this way, Eq. (10) reduces to a form similar to the 2D nonlinear PSE discussed in Chang [2]. The major drawback of this approach is that a Fourier decomposition for the mean flow must be done with a proper choice of the fundamental spanwise wave number that is commensurate with the disturbance wave number of interest. The disturbance decomposition can only be constructed based on the same Fourier series. Even a linear problem would require solutions of the nonlinear PSE for this window PSE approach. Alternatively, the disturbance plane may be formulated in physical space; however, doing so would require more grid points on the $(y, z)$ plane to resolve the disturbance structure. The quasi-parallel eigenvalue solution at a given streamwise location may be used as the initial condition for the PSE 
marching. However, it should be noted that such parallel initial condition would result in transient effects on the marching solutions.

The plane-marching PSE approach is rather time-consuming despite the fact that it is a straightforward extension of the 2D PSE approach. Generation of the initial disturbance mode alone via a $2 \mathrm{D}$ eigenvalue solution alone would take significant computational time. It is therefore not suitable for routine engineering calculations for $3 \mathrm{D}$ boundary layers. Hu and Zhong [19] used the spectral collocation method to solve a form of governing equations similar to Eq. (10) for a 2D stability problem with periodic spanwise boundary conditions. Extensive studies on the planemarching PSE would require more general boundary treatments on the cross plane. The plane-marching PSE method has not been implemented in LASTRAC at this moment.

\section{B. Surface-Marching PSE}

Instead of solving a plane full of disturbances simultaneously, in this approach, we look for a local PSE solution for a single surface-normal line. This local solution is made possible by evaluating $x$ - and $z$-derivatives in Eq. (10) by using only neighboring surface points. By accounting for both derivatives on the body surface, we hope to include the effects of the mean flow and disturbance variation in $x$ and $z$ simultaneously. The concept is quite similar to the formulation for numerical solutions of the 3D boundary layer equations in which viscous terms in both $x$ and $z$ directions are dropped by orders of magnitude considerations and the governing equations are rendered parabolic along the major flow direction. The marching solutions can be performed along any direction on the body surface as long as the point under consideration falls within the zone of dependence formed by the known solutions given at neighboring points (see Wang [18]). The concept of zone of influence and dependence comes from characteristic theory for the simplified boundary layer equations. For a given point on the 3D surface, the zone of dependence is the wedge-shaped region formed by the intersecting inviscid and surface limiting streamlines. Typically, numerical solutions of the 3D boundary layer equations are obtained by marching on the 3D surface along both streamwise $(x)$ and spanwise (z) directions (e.g., BL3D code [20]).

To account for a more general disturbance on a 3D surface, we modify the disturbance form, Eq. (9), to

$$
\psi_{m}=\tilde{\psi}_{m}(x, y, z) e^{i\left(\int_{x_{0}}^{x} \alpha(\xi, z) d \xi+\int_{z_{0}}^{z} \beta(x, \eta) d \eta\right)}
$$

where a complex wave number $\beta=\beta(x, z)$ is introduced in addition to $\alpha=\alpha(x, z)$. The exponential part of Eq. (11) can be generalized to $e^{i \theta}$. The wave numbers then are defined by $\alpha=\partial \theta / \partial x$ and $\beta=\partial \theta / \partial z$, respectively. Mack [21] pointed out that within the framework of conservative wave theory, these two wave numbers are not entirely independent. To keep the wave front potential $\theta$ intact, the following irrotational relation must be satisfied:

$$
\frac{\partial \alpha}{\partial z}=\frac{\partial \beta}{\partial x}
$$

For 2D or infinite swept wing boundary layers, a constant and real spanwise wave number satisfies the above relation because the streamwise wave number is independent of $z$.

The governing equation takes a form similar to Eq. (10), a set of nearly parabolic equations. Similar to the 2D PSE, the weak ellipticity associated with the pressure shape function gradient remains. A procedure similar to $3 \mathrm{D}$ boundary layer equations may be used to solve Eq. (10) over a rectangular domain on the surface provided proper initial conditions are given. Figure 2 depicts examples of the domain on a tapered wing surface. Unlike the case of a line-marching PSE method to be discussed later, the spanwise coordinate is fixed by the selection of the spanwise boundary line parallel to the leading edge. As shown in the figure, we need initial conditions on two surface lines: one parallel to the leading edge $\left(x=x_{0}\right)$ and one roughly aligned with the free-stream direction $\left(z=z_{0}\right)$. The use of a nonorthogonal coordinate system offers a more flexible choice of initial condition lines. Both streamwise and spanwise wave numbers, $\alpha$ and $\beta$, are complex in general. Herbert [9] suggested that two normalization conditions derived from the kinetic energy norm can be used to determine these two wave numbers, $\alpha$ and $\beta$. This approach has two drawbacks. Firstly, the irrotational relation Eq. (12) may not be satisfied. In addition, our numerical experiments 
indicate that while this works for a weakly $3 \mathrm{D}$ boundary layer, two normalization conditions may compete with each other and result in divergence during the wave number iteration procedure for moderate to strong spanwise mean flow variations.

Alternatively, we may use only the normalization condition in $x$ to determine $\alpha$ and then impose Eq. (12) to evaluate $\beta$. This procedure implies that any change in the streamwise growth rate (determined by the imaginary part of $\alpha$ ) along the spanwise direction will incur a non-zero imaginary part of $\beta$. For a moderately $3 \mathrm{D}$ boundary layer, the disturbance wave number (and its growth or decay) will change substantially during the PSE marching. This change results in numerical difficulties and leads to extremely large, unphysical growth or decay of the disturbance. Alternatively, we may require that only the real part of $\beta$ be evaluated by Eq. (12), i.e.,

$$
\frac{\partial \alpha_{r}}{\partial z}=\frac{\partial \beta_{r}}{\partial x}
$$

The imaginary part of $\beta$ is set to zero. This approach should not reduce numerical accuracy because the shape function dependency on $z$ can account for any disturbance growth (or decay) and the irrotational wave front is preserved by the real part of $\beta$. Although not satisfying the wave front potential relation, a real constant $\beta$ is also used in the 3D PSE marching procedure. As long as a large portion of the wave behavior is incorporated in $\beta$, additional phase or amplitude variation can be resolved by the shape function variation in $z$. Strictly speaking, the irrotational property of the wave vector, Eq. (12), is only valid for the wave form determined by the wave number norm chosen within the context of a nonparallel boundary layer. Additional shape function variations in both $x$ and $z$ would alter the wave front for different flow disturbances and for different wall-normal locations. Satisfying Eq. (12) is thus not a critical requirement for nonparallel boundary layers with strong mean flow variations in both surface directions. Further validations against DNS results are necessary to determine the merit of the different approaches discussed above.

The surface marching procedure described above can account for disturbance evolution on the entire body surface in a consistent manner. However, it remains to be determined to what extent the marching solution is influenced by the initial conditions. In this research, we impose a local eigenvalue solution at $\left(x_{0}, z_{0}\right)$. A marching PSE solution is then obtained by neglecting the spanwise changes for the initial line that roughly aligns with the free stream (see Fig. 2). For the initial line nearly parallel to the leading edge, we impose the same eigenfunction obtained at $\left(x_{0}, z_{0}\right)$. In general, this combination of initial conditions would result in transient effects for the marching solutions. However, the wave number iterations for $\alpha$ may damp out transient effects rapidly and limit the transient effects on the growth rate computed in the vicinity of the initial lines. The dependency of initial conditions that spans over a substantial part of the domain is the major drawback of the surface-marching PSE approach. Obtaining initial conditions from a higher-fidelity tool or advanced receptivity theories would help relieve this stringent requirement. Alternatively, a local line-marching PSE procedure with additional approximations can be used, which will be discussed in the following section.

\section{Local Line-Marching PSE}

In this approach, instead of solving for the disturbance evolution over a rectangular region on the surface, PSE marching is performed locally along a pre-identified line path on the surface. Along this line, a local PSE solution is solved for with the effects of spanwise mean flow variation being accounted for or neglected entirely. The main idea is to identify a path (e.g. streamline or group velocity line) that has relatively weak spanwise effects such that a local PSE solution represents most of the disturbance evolution. Governing equations and wave decomposition are similar to Eqs. (10) and (11), respectively. The spanwise wave number $\beta$ is assumed to be real. By neglecting viscous second derivatives along $z$, coupled discretized PSE solutions at two neighboring spanwise wall-normal lines are solved simultaneously. This numerical technique is similar to the one used for the local nonparallel eigenvalue solution in a nonparallel boundary layer (see [2]). A real $\beta$ does not pose any restriction because as in the surfacemarching PSE, additional phase and amplitude modulations associated with the spanwise mean flow variation are captured in the shape function variation in $z$. Two separate norms in $x$ are used to determine two streamwise wave numbers. Similar to the surface-marching case, a norm in $z$ to determine a complex $\beta$ results in a competing situation and wave number iterations would fail to converge. Mean flow variations along both directions on the surface are 
accounted for in this procedure. The inviscid streamline is usually used as the integration path because the group velocity line requires a separate quasi-parallel linear stability solution. The spanwise $(z)$ coordinate is aligned with a direction in which the mean flow variation is weak. The isobars seem to be a logical choice but it takes extra calculations to identify them. Numerical experiments indicate that the conical direction formed by the leading edge and trailing edge of a swept wing works fine for most cases.

When a "good" spanwise direction is used, the local line-marching method may be further simplified by neglecting the = variation locally. This simplification is justified only as an engineering practice. Nonetheless, for many practical applications. this simplified local line-marching (without accounting for spanwise mean flow variations) offers a satisfactory solution that agrees well with those from the original local line-marching or the surfacemarching approach. For example, in a tapered wing boundary layer, the conical ray direction may be used as the spanwise direction along which the mean flow variation is almost negligible. This $z$ direction varies locally at each marching location and may be treated easily within the framework of a nonorthogonal coordinate system. For practical applications, the local line-marching method may be used for transition correlations due to its numerical efficiency.

\section{Quasi-parallel LST}

By further imposing the locally parallel assumption for the governing equations, an eigenvalue problem may be formed locally for a given disturbance frequency and complex spanwise wave number. In the saddle point method [3, 22], $\mathrm{N}$-factor integration is performed along the group velocity direction obtained by solving the dispersion relation in conjunction with the requirement that $d \alpha / d \beta$ is real. To close the problem, it is further assumed that the disturbance growth rate is maximal along the group velocity direction. The spanwise wave number $\beta$ is complex and varying along the marching direction in this case. However, the $\mathrm{N}$-factor integration does not take into account the spanwise growth (or decay). Besides the saddle point method, the $\mathrm{N}$-factor can be calculated by integrating the most unstable disturbance along the inviscid streamline. In many cases, there is little distinction in the $\mathrm{N}$-factor contours between the saddle point and streamline maximization procedures. Both approaches are implemented in the LASTRAC.3d code.

\section{Mean Flow Computation}

Depending on the complexity of the 3D boundary layer configurations, various methods may be used to compute the mean flow. For a tapered wing, the conical assumption can be used to simplify the boundary layer equations (see [16]). Under this assumption, a similarity solution as a function of the conical ray angle and the wall-normal distance exists. Solutions at different spanwise locations can be constructed according to the conical radius measured from the origin of the rays formed by the leading edge and trailing edge to the location of interest. The boundary layer code BLSTA[16] is used to generate the transonic tapered wing case discussed in the next section.

Boundary layer solutions for full 3D cases require a marching solution similar to the one discussed previously for the 3D surface-marching PSE procedure. Given the inviscid solution on the body surface, the boundary layer equations are solved consecutively via marching along both streamwise and spanwise directions on the body surface. The boundary layer code BL3D [20] is used to obtain 3D boundary layer solutions for the present study. The wall-normal velocity is neglected in the stability calculations because this velocity component as computed by BL3D has not been validated. Navier-Stokes solutions, of course, may be used as an alternative.

\section{Results and Discussion}

The nonorthogonal coordinate system used in LASTRAC offers flexible choices of streamwise and spanwise directions. The $\mathrm{N}$-factor integration in any local line-marching methods as discussed previously may be performed via marching along the chordwise (streamwise) surface grid line, the inviscid streamline, or the group velocity directions. In the grid line or streamline marching, a real the wave number $\beta$ is used and disturbance growth along the spanwise direction is either accounted for in the shape function variation or completely neglected as an engineering approach. Three spanwise directions are available in LASTRAC: along the spanwise grid line, the conical direction, or perpendicular to the inviscid streamline. For a tapered wing, the first two options are naturally suitable because the spanwise mean flow variation along that direction is usually small. The last option is acceptable 
downstream of the leading edge but would result in large errors near the leading edge where the streamline normal is almost perpendicular to the leading edge. For forebody configurations such as a circular or elliptic cone, the azimuthal direction is perhaps a good choice for $\beta$. An arbitrary combination of the marching and spanwise directions may be employed for a line-marching PSE approach

The line-marching PSE method provides a localized solution at the given marching path. The $\mathrm{N}$-factor distribution is determined along the given path and any history effect due to spanwise variations is neglected. The surfacemarching PSE method, on the other hand, can be used to obtain entire surface distribution of N-factors. Both linemarching and surface-marching PSE methods are implemented in the LASTRAC code. In the following discussion, we first validate the nonorthogonal coordinate system by comparing its results with existing validated orthogonal solutions. Then N-factor calculations using LST or various PSE methods are presented for several 3D boundary layer configurations. All calculations presented herein are performed by accounting for the body curvature along both chordwise and spanwise directions.

\section{A. Validation of the Nonorthogonal Coordinate System}

To validate the nonorthogonal coordinate system and governing equations, we calculate the stationary crossflow instability wave over an incompressible infinite swept wing boundary layer by using several combinations of marching and spanwise directions. As shown in Fig. 3, three marching directions are identified: along the chord normal $\left(x_{0}\right)$, along the inviscid streamline $\left(x_{1}\right)$, and along the free stream $\left(x_{2}\right)$. In the following calculations, the spanwise wavelength is fixed for two spanwise directions: parallel to the leading edge $\left(z_{0}\right)$ and along the direction normal to the inviscid streamline $\left(z_{1}\right)$.

The quasi-3D solution computed by the earlier release of LASTRAC via marching along the chord normal direction with an orthogonal coordinate system (i.e. the $\left(x_{0}, z_{0}\right)$ coordinate) is used as the baseline. It is considered the "correct" solution because the governing equations under an orthogonal coordinate are well-suited for an infinite swept wing. Numerical solutions using this orthogonal coordinate system have been validated against direct numerical simulations and excellent agreement was achieved [15]. Three additional combinations were investigated: $\left(x_{2}, z_{0}\right),\left(x_{1}, z_{0}\right)$, and $\left(x_{1}, z_{1}\right)$. They are denoted by I, II, and III, respectively. Figure 4 shows the resulting nonparallel (PSE) N-factors based on disturbance kinetic energy for a spanwise wavelength of $10 \mathrm{~mm}$ (which is the most unstable stationary crossflow disturbance). We compare $\mathrm{N}$-factors instead of growth rates because the former is an invariant under coordinate transformation while the latter is not. Both cases I and II give excellent agreement with the baseline solution. On the other hand, marching along the streamline with a normal spanwise coordinate (case III) gives an incorrect solution. This result is not surprising because case III differs from the others where the spanwise direction is consistent with the baseline solution. Although not shown here, quasiparallel LST calculations using various coordinates produce identical results.

The above comparisons indicate that a streamline or free-stream direction marching with a spanwise coordinate parallel to the leading edge gives a correct solution and the streamline orthogonal marching (case III) should not be used in a 3D boundary layer. We can also calculate the nonparallel $\mathrm{N}$-factors by using the surface-marching PSE method. Figure 5 shows the resulting $\mathrm{N}$-factor contours on the wing surface for a rectangular nonorthogonal domain. The spanwise direction is aligned with the spanwise domain boundary (parallel to the leading edge). The $\mathrm{N}$-factor contours are parallel to the leading edge for the infinite swept-wing surface as expected. The $\mathrm{N}$ values along a chord-normal cut are also plotted in Fig. 4 and excellent agreement with the baseline solution is evident.

\section{B. Tapered Wing boundary Layer}

A tapered wing boundary layer provides a more realistic example for the study of $3 \mathrm{D}$ effects. Unlike an infinite swept wing, the mean flow in a tapered wing boundary layer varies along the spanwise direction. The mean flow is calculated by using the BLSTA code (Wie [16]) using the conical assumption, i.e., the mean flow does not vary along a ray originating from the point formed by the intersection of the leading and trailing edges. The mean flow is computed with an orthogonal coordinate system where marching is performed along a circular arc orthogonal to 
both leading and trailing edges. Stability calculations could also be formulated by a similar conical assumption. However, we did not pursue this approach and instead use a more general nonorthogonal $3 \mathrm{D}$ framework in this study.

A Mach 0.8 flow over a tapered wing was calculated. The leading and trailing edge sweep angles are $28^{\circ}$ and $14^{\circ}$, respectively. The surface grid for mean flow calculations is shown in Fig. 6. Also shown in the figure are three different marching paths formed by tracing a gridline along the streamwise direction, along the inviscid streamline and along the group velocity direction. We perform local line-marching PSE calculations for the former two marching paths with a conical spanwise coordinate varying locally at each location of the tapered wing. Figure 7 shows the corresponding linear PSE solutions obtained for the most amplified traveling crossflow mode with a frequency of $1.5 \mathrm{kHz}$ and a spanwise wavelength of $12 \mathrm{~mm}$. Evidently, both marching paths give identical solutions and may be used for tapered wing stability calculations.

Quasi-parallel LST calculations were also performed by maximizing the disturbance growth rate and integrating Nfactors along the inviscid streamline. Figure 8 shows the resulting $\mathrm{N}$-factor along with those calculated by using the conventional saddle point method along the group velocity line. Both methods give almost identical $\mathrm{N}$ values except at small $x / c$. For a swept wing boundary layer, maximizing growth rates along the inviscid streamline tend to give very close $\mathrm{N}$-factor results with the traditional saddle point method.

Surface-marching PSE calculations were also performed for this configuration. The resulting $\mathrm{N}$-factor distribution, as shown in Fig. 9(a), roughly scales according to the Reynolds number (i.e., the distance from the leading edge). Figure 9(b) depicts $\mathrm{N}$-factor distributions at various spanwise locations. For comparison, the $\mathrm{N}$-factors obtained by the local line-marching PSE solutions are also shown in the figure. The agreement between these two PSE marching solutions is very good. The small discrepancy is mainly due to the small difference in the initial conditions. Although not shown here, spanwise disturbance correction in the local line-marching PSE calculation was also performed for the above traveling crossflow mode. It was found that for the present case, the correction is negligible.

The above results indicate that the local line-marching or surface-marching PSE method gives almost identical solutions for the test case. Any of these PSE methods may be used as an alternative to LST for instability wave and transition calculations for a tapered wing boundary layer in order to more accurately account for the nonparallel and surface curvature effects.

\section{Supersonic Finite Swept Wing}

The next test case is a Mach 2 flow over a 56-degree finite swept wing. The computed surface $C p$ distribution and wing geometry are shown in Fig. 10(a). Except near the leading edge, the pressure distribution indicates substantial 3D variations. The computed crossflow Reynolds number contours are shown in Fig. 10(b). The maximum $R_{c f}$ is around 1500. The inboard side tends to have larger crossflow Reynolds numbers. The engulfed negative crossflow Reynolds number region in the middle is associated with the pressure variation in that region. The BL3D code reads in the inviscid solution and finds the attachment line location near the leading edge. An approximated similarity solution is then calculated at the attachment line. Using this solution as an initial condition, the boundary layer profiles are computed over the entire surface.

We do not intend to perform exhaustive transition prediction calculations for this configuration. Instead, we will investigate 3D disturbance evolution for the most amplified traveling crossflow mode on the wing surface. From quasi-parallel LST calculations, the most amplified mode has a frequency of $6 \mathrm{kHz}$ and a spanwise wavelength of 16 $\mathrm{mm}$. We first performed surface-marching PSE calculations. A local nonparallel eigenvalue calculation around one of the leading edge corners was performed for the most unstable mode by neglecting the spanwise mean flow variations. The computed streamwise wave number, $\alpha$ and eigenfunctions were then imposed as initial conditions along a line parallel to the leading edge. The initial conditions along the inboard chordwise line were obtained by a local line-marching PSE calculation. While the chordwise initial conditions are a reasonable approximation, the ones along the spanwise direction are certainly not in light of the strong mean flow variation from inboard towards the outboard direction. Better initial conditions might be obtained by using local nonparallel eigensolutions at each location along the spanwise initial line. 
We first assess the effects of initial conditions. Figures 11 (a) and 11(b) show the $\mathrm{N}$-factor contours evaluated by the peak chordwise velocity perturbations for two calculations using the local nonparallel eigensolutions obtained at inboard and outboard corners, respectively. Although both plots show similar trends, they differ in details. The difference in these results illustrates the dependency on initial conditions for the surface-marching PSE approach.

In the above calculations, we imposed a constant spanwise wave number $\beta$. As discussed in the previous section, a complex spanwise wave number can also be imposed and updated during the surface-marching procedure according to the irrotational phase condition, Eq. (12). To maintain numerical stability, a real irrotational condition, Eq. (13) may also be used. The use of the additional constraint on the spanwise wave number implies that the modal wave number may vary downstream of the marching. This can be seen in Fig. 12(a)-12(c) where the real parts of the effective spanwise wave number (after accounting for the shape function variation along the spanwise direction) contours evaluated at the peak chordwise velocity locations are plotted for real constant $\beta$, complex $\beta$ using Eq. (12), and real $\beta$ using Eq. (13), respectively. The additional constraint causes the effective wave number to vary significantly from the mid-chord region on and extend all the way to the trailing edge. It appears to introduce additional length scales to the disturbance field. The corresponding $\mathrm{N}$-factor contours for the latter two cases shown in Fig. 12 are plotted in Figs. 13(a) and 13(b). Compared to Fig. 11(b) that uses the same initial conditions, these results reveal more complex structures and very large $\mathrm{N}$ values are observed within the "pockets" near the trailing edge. Although the appearance of multiple length scales in the disturbance evolution might look unphysical, further validations against DNS are necessary to judge the merits of imposing the irrotational condition.

The local line-marching PSE method was also used to compute the above case. The same inboard corner initial conditions given for Fig. 11(a) were used for the local line-marching calculations. The spanwise mean flow and disturbance variations can be either accounted for by solving two wall-normal lines simultaneously or neglected as an approximation. The marching calculation was performed along the streamwise (chordwise) gridline direction with a spanwise coordinate aligned with conical rays. Figures $14(\mathrm{a})-14(\mathrm{c})$ depicts the $\mathrm{N}$-factor contours evaluated by the total disturbance kinetic energy for the surface-marching, local line-marching with spanwise corrections, and local line-marching without spanwise corrections, respectively. Despite differences in details, the overall agreement of the three methods is acceptable. A close-up comparison reveals an $\mathrm{N}$-factor difference of about 1 or 2 . It must be cautioned that these comparisons are for a single disturbance mode. The local line-marching PSE method tends to give consistent trends with the surface-marching method. Because a transition correlation based on $\mathrm{N}$-factor is empirical in nature, it remains to be answered that whether the surface-marching method offers a better prediction tool.

\section{Axisymmetric Cone at an Angle of Attack}

Besides swept wing boundary layers, axisymmetric or elliptic cones at an angle of attack represent another group of 3D boundary layers. We investigate a Mach 3.5 flow over a 5-degree half-angle cone with a 4-degree angle of attack. Boundary layer transition on this configuration has previously been investigated experimentally by King [17]. The mean flow was computed by using the CFL3D code with a $256 \times 33 \times 177$ grid along the streamwise, azimuthal, and wall-normal directions, respectively. Figure 15 depicts the surface mesh and computed inviscid streamlines. The maximum crossflow Reynolds number is about 1600.

We first performed linear PSE calculations along two different paths as shown in Fig. 16. The gridline path is roughly aligned with the ray of the cone generator. A spanwise wavelength of $1 \mathrm{~mm}$ (which corresponds to the most amplified stationary crossflow mode) was fixed along the azimuthal direction for both cases. Figure 17 shows the resulting nonparallel stationary crossflow instability $\mathrm{N}$-factors (computed by a local line-marching PSE without spanwise corrections) measured by the disturbance kinetic energy. The large $\mathrm{N}$ values indicate that the flow is very unstable. Unlike the previous swept wing examples, two different marching paths give a difference in $\mathrm{N}$-factor of about 5. A more comprehensive study is necessary to judge the superiority of either marching path. Previous results seem to indicate that marching along the inviscid streamline in general gives better transition correlation while marching along the ray usually overpredicts the $\mathrm{N}$ value.

The above PSE results were obtained by neglecting the spanwise (azimuthal) mean flow and mode shape variation. Figure 18 shows linear PSE results obtained by streamline marching with and without accounting for the spanwise variation. As shown in the figure, the $3 \mathrm{D}$ effects result in a smaller $\mathrm{N}$-factor and the difference is slightly larger than 
1. In the linear PSE calculation with spanwise correction, the effective spanwise wave number becomes complex because the shape function is allowed to vary in the spanwise direction. The maximum spanwise wavelength correction is only about 2 percent. It appears that the disturbance is decaying along the spanwise direction (from the windward pointing towards the leeward direction), which is expected because of the decrease of crossflow strength along that direction. Due to the lack of resolution along the azimuthal direction and large mean flow gradient near the apex of the cone, the surface-marching PSE method gives solutions that possess strong and unphysical transient effects near the leading edge. A better resolved mean flow and more carefully treated initial conditions are necessary for further studies.

We also performed transition correlation for stationary crossflow instability by maximizing the growth rate with respect to spanwise wave number by using the conventional LST approach. The streamline marching path was used for integration. Figure 19 shows the location where the $\mathrm{N}$ value first reaches 10 for each streamline. The results show that transition location moves upstream near the center plane. Both windward and leeward planes are very stable to stationary crossflow disturbance. These results seem to be consistent with previous investigations (Malik and Balakumar [5]).

\section{Conclusion}

Advanced transition prediction methods based on traditional and the state-of-the-art PSE methods are studied for 3D boundary layers. Numerical formulations of various transition prediction methods for general 3D boundary layers are discussed. The surface-marching and local line-marching PSE approaches provide a means of calculating disturbance evolution in 3D boundary layers beyond the traditional quasi-parallel LST methods. The use of a nonorthogonal coordinate system facilitates easy adaptation to the mean flow grid and flexibility in choosing the combination of spanwise and marching directions. These 3D transition prediction methods are implemented in the new release of the LASTRAC code.

Several test cases that cover a range of speed regimes are studied by using the surface-marching and line-marching PSE methods. The results indicate that both methods can capture 3D effects due to mean flow variations. For a tapered wing boundary layer, the line-marching PSE with a conical spanwise direction gives identical solutions to that by the surface-marching method. For the supersonic swept wing case, initial conditions have noticeable influence on the computed $\mathrm{N}$-factor contours. However, with the same initial conditions, line-marching and surfacemarching PSE methods give qualitatively similar but quantitatively different solutions in $\mathrm{N}$-factors. Further validations against existing data or DNS results are necessary to assess the merits of each approach.

\section{Acknowledgments}

The author would like to thank Dr. Meelan Choudhari of Langley Research Center for computing the NavierStokes mean flow for the cone case. Fruitful discussions with Drs. Fei Li and Venkit lyer about the 3D boundary layer solutions are also acknowledged.

\section{References}

1. Wlezien, R. W. and Veitch, L., "Quiet Supersonic Platform Program Advanced Algorithms for Design and Optimization of Quiet Supersonic Platforms." AIAA Paper 2002-0143, 2002.

2. Chang, C.-L., "The Langley Stability and Transition Analysis Code (LASTRAC): LST, Linear and Nonlinear PSE for 2-D, Axisymmetric, and Infinite Swept Wing Boundary Layers," AIAA Paper 2003$0974,2003$.

3. Nayfeh, A. H., "Stability of Three-Dimensional Boundary Layers," AIAA J., Vol. 18, pp. 406-416, 1980.

4. Cebeci, T. and Stewartson, K., "On Stability and Transition of Three-Dimensional Flows," AlAA J., Vol. 18 , pp. 398-405, 1980. 
5. Malik, M. R. and Balakumar, P., "Instability and Transition in Three-Dimensional Supersonic Boundary Layers," AIAA Paper 1992-5049, 1992.

6. Atobe, T. and Chen, H. H., "Stability and Transition Analysis of NEXST-1 NLF Wing Model at Mach 2," AIAA Paper 2003-0971, 2003.

7. Spalart, P. R. and Watmuff, U. H., "Experimental and Numerical Study of Turbulent Boundary Layer with Pressure Gradients," J. Fluid Mech., Vol. 249, pp.227-371, 1993.

8. Guo, Y., Adams, N. A., and Kleiser, L., "Direct Numerical Simulation of Transition in a Spatially Growing Compressible Boundary Layer using a New Fourier Method," Direct and Large-Eddy Simulation I, eds. P. R. Voke, L. Kleiser, J. P. Chollet, Kluer, London, pp. 249-250, 1994.

9. Herbert, Th., "On the Stability of 3D Boundary Layers," AIAA Paper 1997-1961, 1997.

10. Aris, R., "Vectors, Tensors, and the Basic Equations of Fluid Mechanics," Prentice-Hall, Englewood Cliffs, NJ. 1962.

11. Streett, C. L., "Direct Harmonic Navier-Stokes Methods for Efficient Simulation of Weakly-Nonlinear Wave Packets," AIAA Paper 1998-0784, 1998.

12. Guo, Y., Malik, M. R., and Chang, C.-L., "A Solution Adaptive Approach for Computation of Linear Waves," AIAA Paper 1997-2072, 1997.

13. Malik, M. R., Li, F., and Chang, C.-L., "Crossflow Disturbances in Three-Dimensional Boundary Layers: Nonlinear Development, Wave Interaction and Secondary Instability," J. Fluid Mech., Vol. 268, pp. 1-36, 1994.

14. Lin, R.-S. and Malik, M. R., "On the Stability of Attachment-Line Boundary-Layers: Part 2, The Effect of Leading-Edge Curvature," J. Fluid Mech., Vol. 333, pp. 125-137, 1997.

15. Li, J., Chang, C.-L., Choudhari, M. and Liu, C., "Cross-Validation of DNS and PSE Results for Instability Wave Propagation in Compressible Boundary Layer past Curvilinear Surfaces," AIAA Paper 2003-3555, 2003.

16. Wie, Y.-S., "BLSTA --- A Boundary Layer Code for Stability Analysis," NASA CR-4481, 1992.

17. King, R. A., "Three-dimensional Boundary-layer Transition on a Cone at Mach 3.5," Experiments in Fluids, Vol. 13, pp.305-314, 1992.

18. Wang, K. C., "On the Determination of the Zones of Influence and Dependence for Three-Dimensional Boundary-Layer Equations," J. Fluid Mech., Vol. 48, p. 397, 1971.

19. Hu, S. H. and Zhong, X., "Nonparallel Stability Analysis of Compressible Boundary Layer using 3-D PSE," ALAA Paper 1999-0813, 1999.

20. Iyer, V.. "Three-Dimensional Boundary-Layer Program (BL3D) for Swept Subsonic of Supersonic Wings with Application to Laminar Flow Control," NASA CR-4531, 1993.

21. Mack, L. M., "Boundary Layer Stability Theory," Special Course on Stability and Transition of Laminar Flow, AGARD Report No. 709, pp. 3/1-81, 1984.

22. Cebeci, T. and Stewartson, L., "On Stability and Transition of Three-Dimensional Flows," AlAA J, Vol. 18, pp. 398-405, 1980.

23. Chang, C.-L., "Langley Stability and Transition Analysis Code (LASTRAC) Version 1.2 User Manual," NASA/TM-2004-213233, June, 2004. 

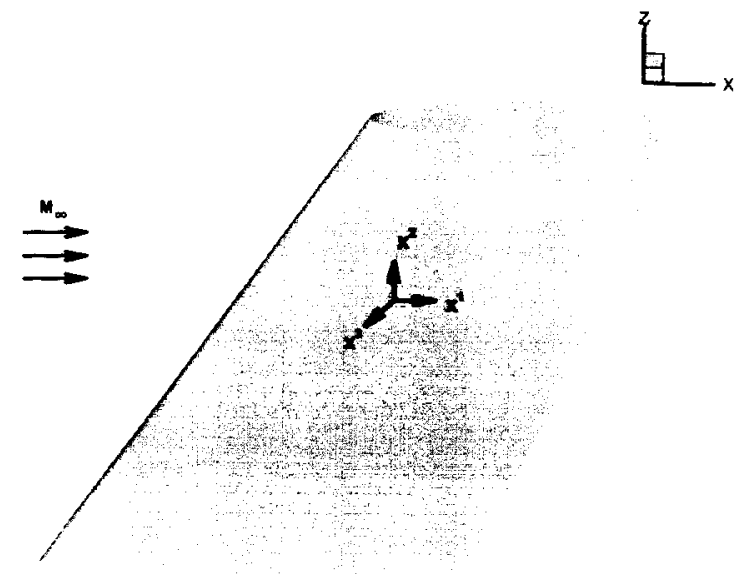

Figure 1: Nonorthogonal body-fitted curvilinear coordinate over a 3D surface.

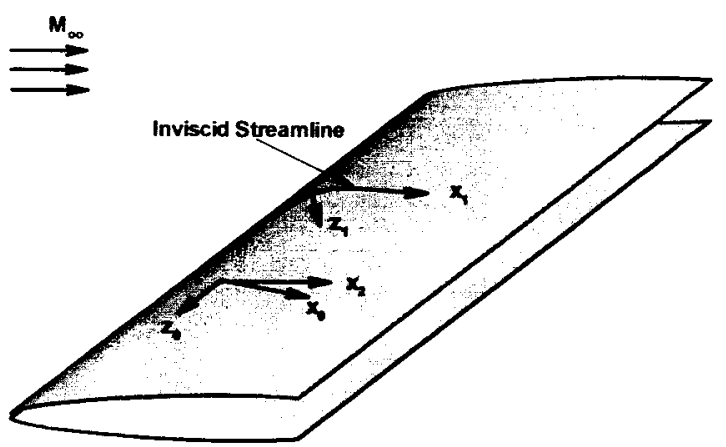

Figure 3: Three different marching coordinates for an infinite swept wing boundary layer.

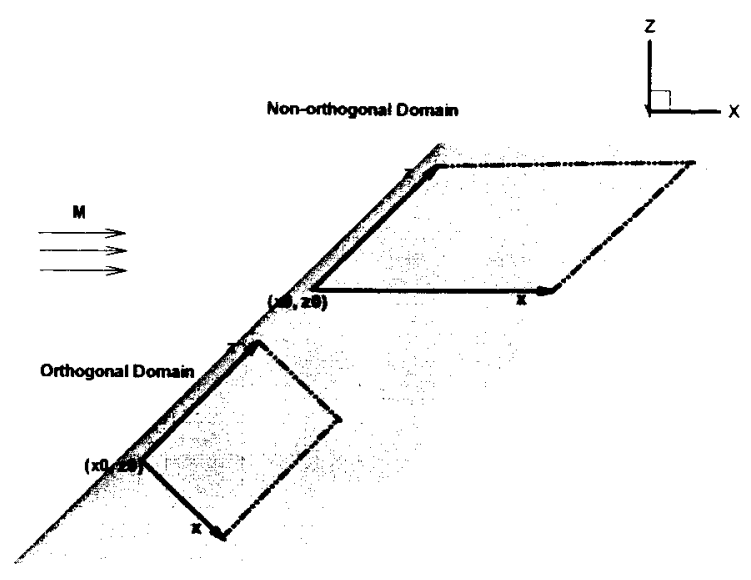

Figure 2: Rectangular domains for surface-marching PSE calculations.

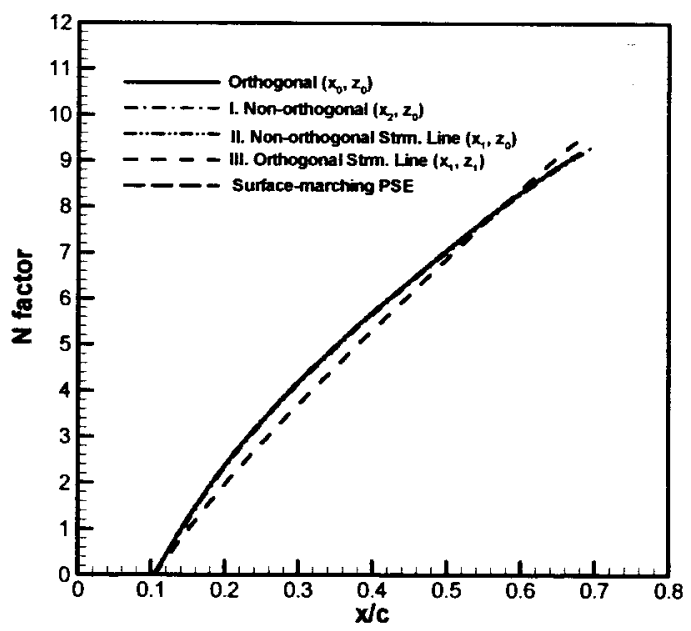

Figure 4: Comparison of PSE N-factors for a stationary crossflow mode using various coordinate systems and surface-marching PSE for an infinite swept wing boundary layer. 


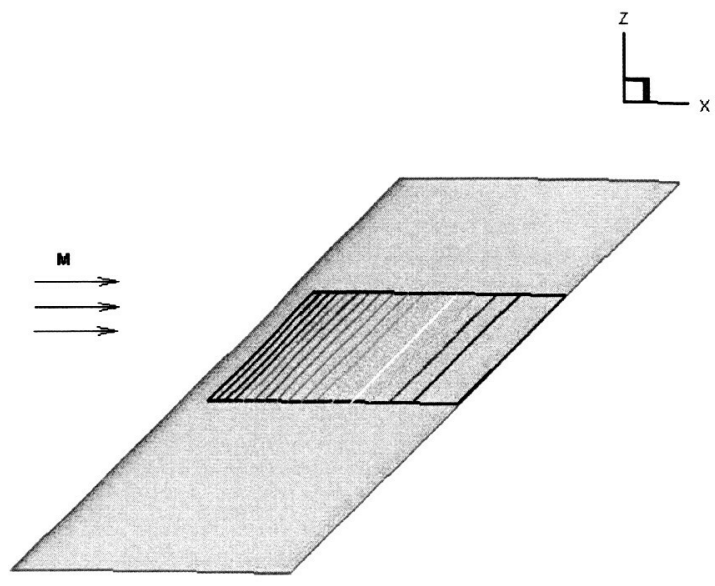

Figure 5: N-factor distribution calculated by surface-marching PSE for the infinite sweptwing boundary layer using a nonorthogonal domain.

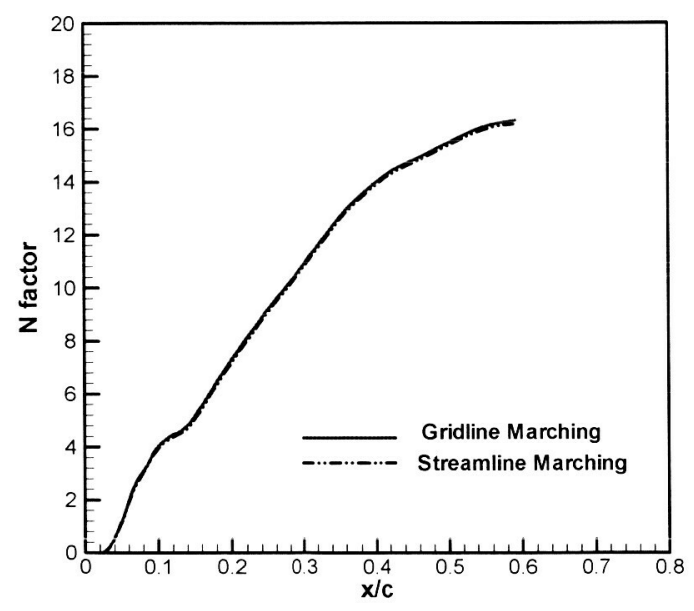

Figure 7: Comparison of linear PSE N-factors for $f=1.5 \mathrm{kHz}$ and $\lambda=12 \mathrm{~mm}$ using two marching paths for a transonic tapered wing boundary layer.

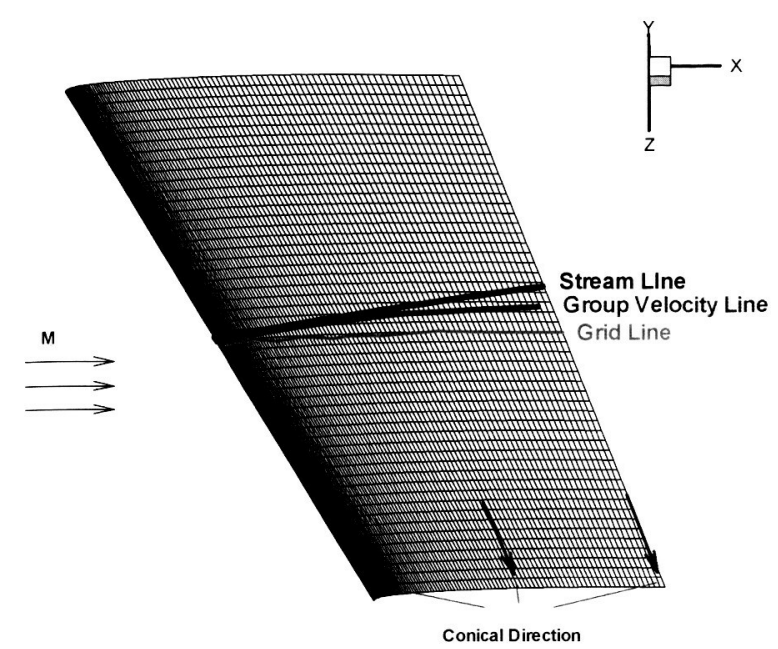

Figure 6: Geometry and surface grid for a transonic tapered wing, showing three marching paths and the conical spanwise coordinate.

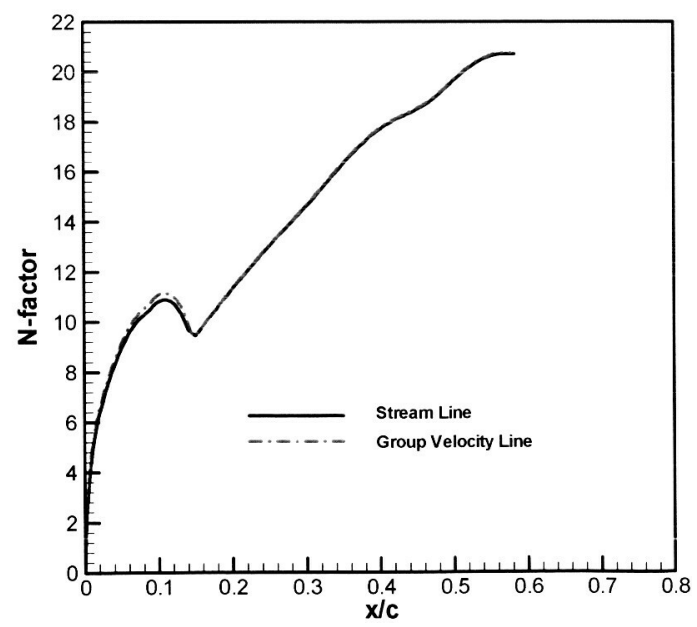

Figure 8: Comparison of maximized LST Nfactors for $f=1.5 \mathrm{kHz}$ using two integration paths for a transonic tapered wing boundary layer. 


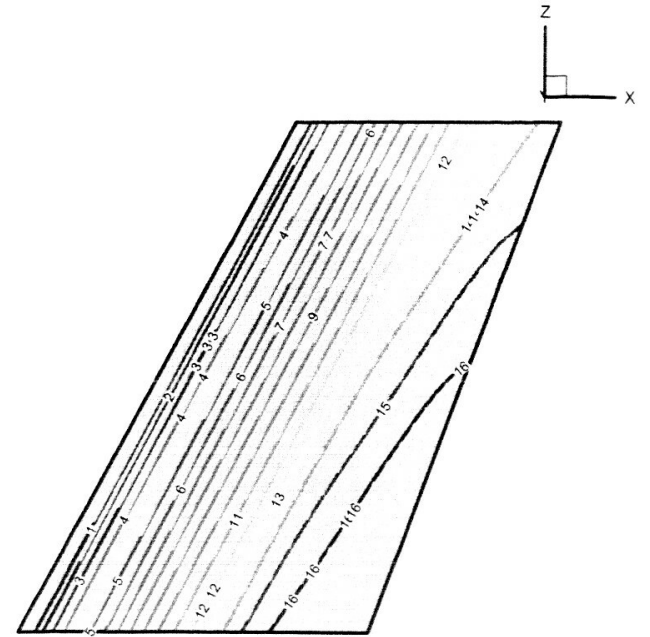

(a)

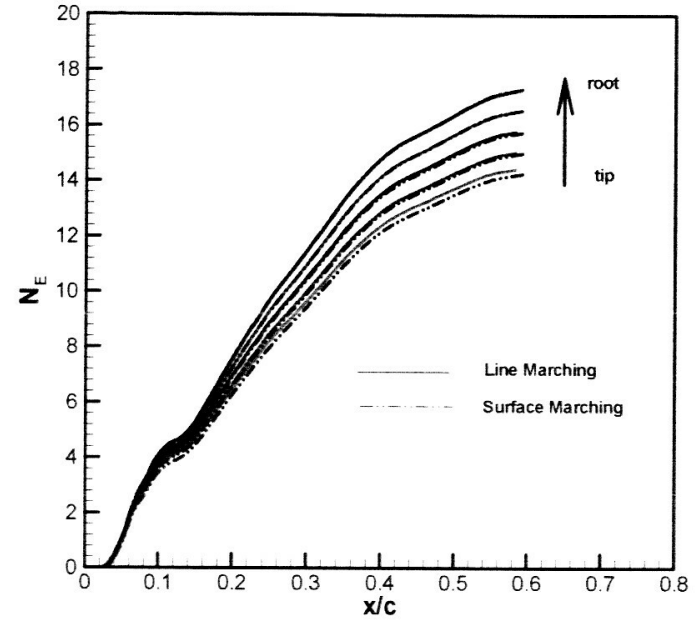

(b)

Figure 9: Nonparallel $\mathrm{N}$-factor (based on disturbance kinetic energy) distribution for $f=1.5 \mathrm{kHz}, \lambda=12 \mathrm{~mm}$ on the transonic tapered wing surface obtained by surfacemarching PSE: (a) $\mathrm{N}$-factor contours on the surface; (b) $\mathrm{N}$-factors from local linemarching at various spanwise locations.
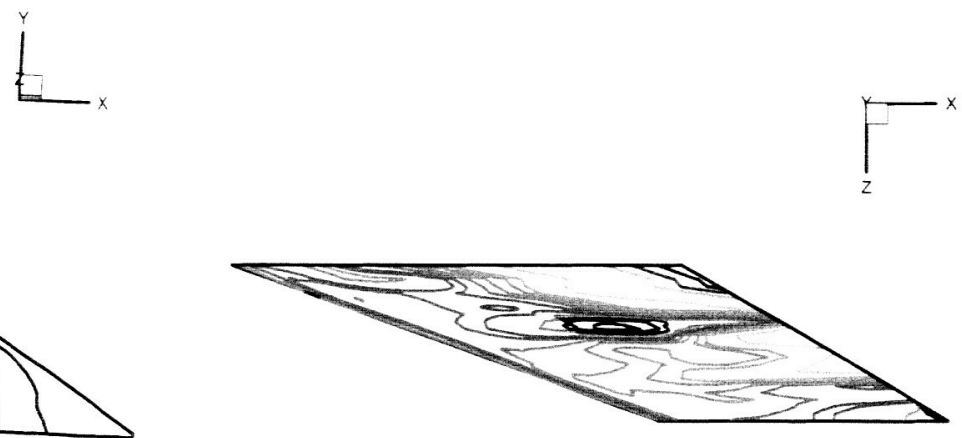

(a)

(b)

Figure 10: Pressure coefficient (a) and crossflow Reynolds number (b) distributions on the surface of a Mach 2, 56-degree finite swept wing 


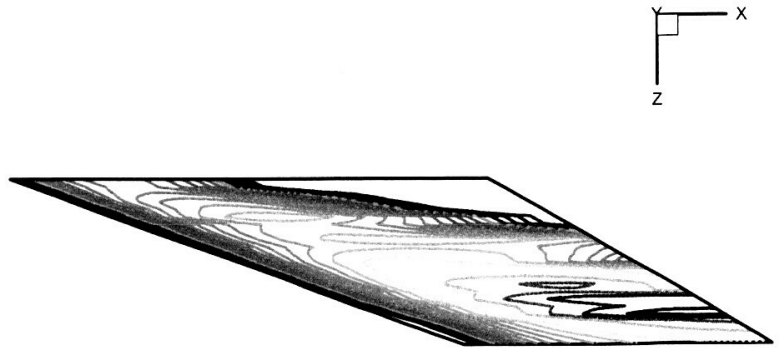

(a)

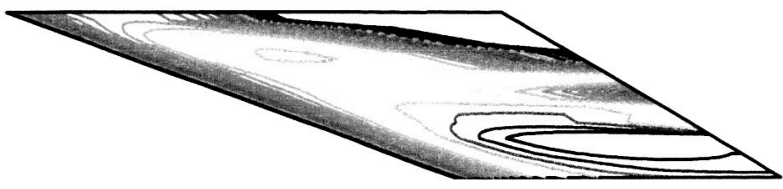

(b)

Figure 11: Surface-marching $\mathrm{N}$-factor contours for $f=6 \mathrm{kHz}$ and $\lambda=16 \mathrm{~mm}$ using two different initial conditions: (a) inboard corner; (b) outboard corner.

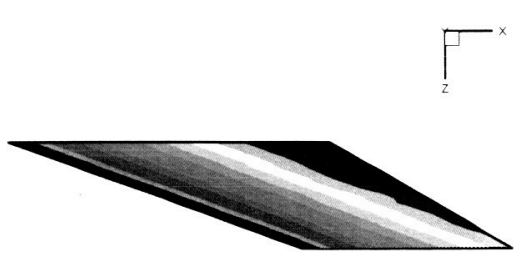

(a)

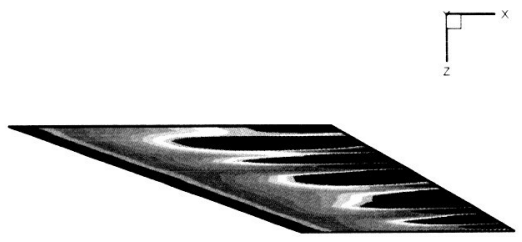

(b)

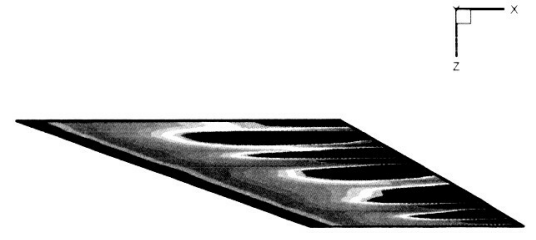

(c)

Figure 12: Real part of the effective spanwise wave number evaluated at peak chordwise velocity locations using: (a) constant real $\beta$; (b) Eq. (12); (c) Eq. (13). 


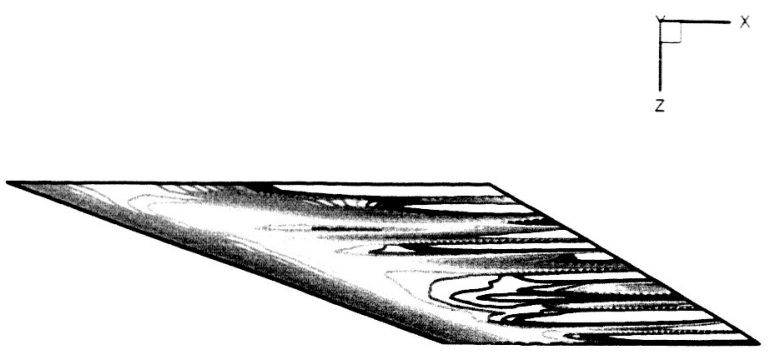

(a)

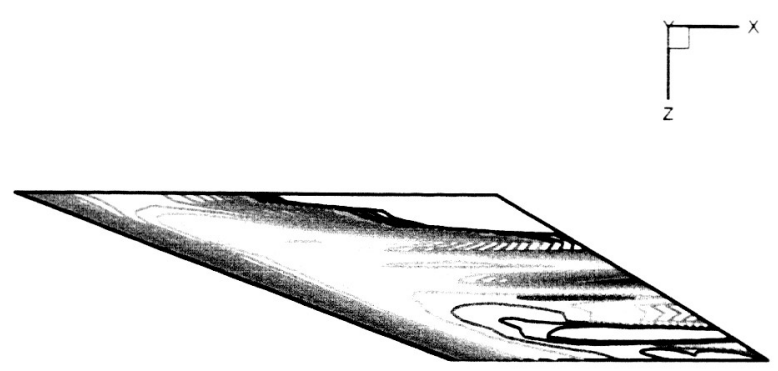

(b)

Figure 13: Surface-marching $\mathrm{N}$-factor evaluated at peak chordwise velocity locations using: (a) Eq. (12); (b) Eq. (13).

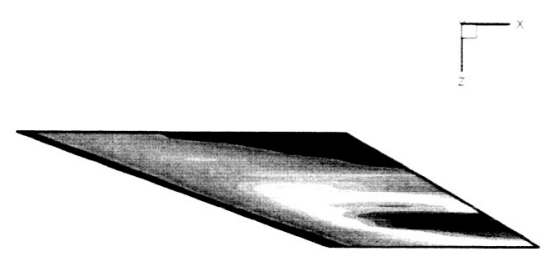

(a)

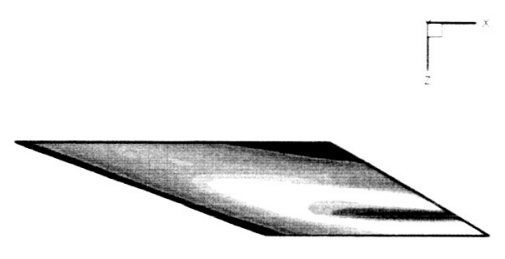

(b)

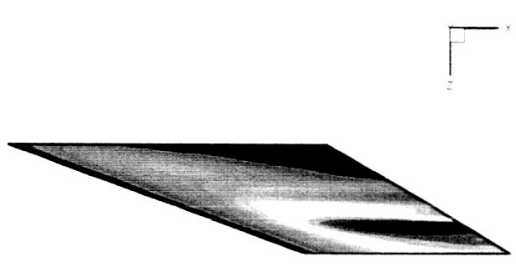

(c)

Figure 14: Comparison of $\mathrm{N}$-factor evaluated by total kinetic energy obtained by (a) surface-marching; (b) local line-marching with spanwise correction; (c) local linemarching without spanwise correction. 


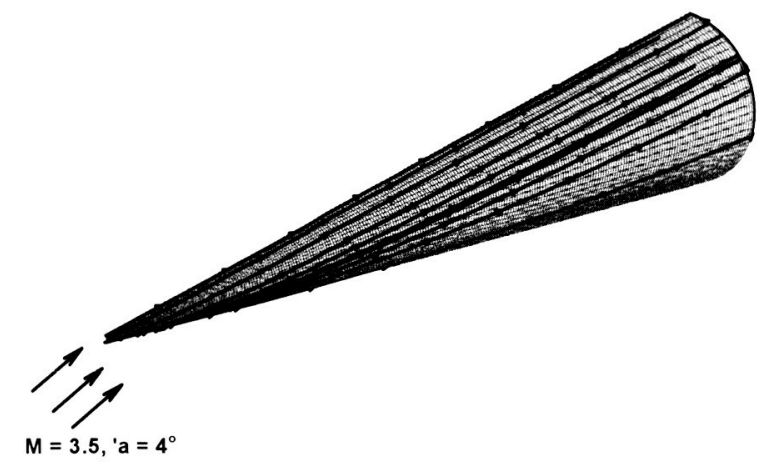

Figure 15: Surface mesh and streamlines for a Mach 3.5 flow over a cone at 4-degree angle of attack.

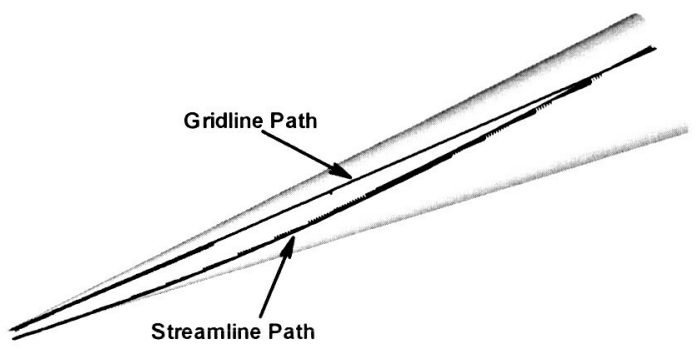

Figure 16: Two marching paths on a Mach 3.5 flow over a cone at 4-degree angle of attack.

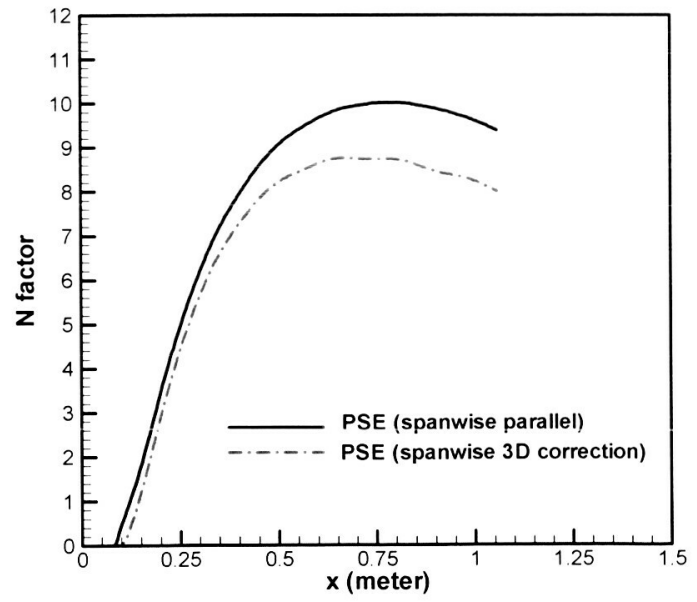

Figure 18: N-factor calculated by linear PSE using local line-marching along the inviscid streamline with and without spanwise mean flow and disturbance correction. 


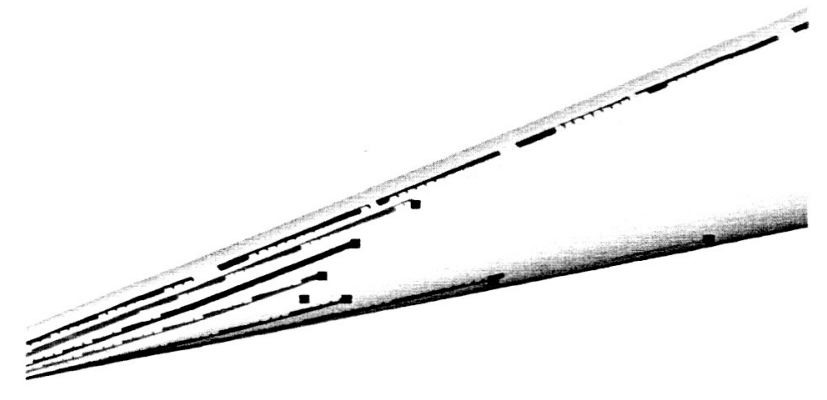

- $\quad N=10$, Stationary Crossflow

Figure 19: Stationary crossflow N-factor distribution calculated by LST marching along the streamline with maximizing the local disturbance growth rates. 